\title{
Interpersonal attachment predicts identification with groups
}

Richard J. Crisp, Claire V. Farrow, Harriet E.S. Rosenthal , Judi Walshd, Jackie Blissette \& Nicola M.K. Penne

We experience many different types of relationship. On the one hand we have relationships with individuals (e.g., a spouse, partner, sister, mother or son). On the other we have relationships with broader, inclusive, and sometimes more abstract, collectives (e.g., a sports team, college society, nationality, or ethnic group). Research programs focusing on these different types of relationship have typically progressed quite independently from one another and have correspondingly specified distinct psychological processes (although for some preliminary integrations see Mikulincer and Shaver, 2001 and Smith et al., 1999). In this article we propose that, under certain conditions, interpersonal and group level affiliations will be related. In particular, we argue that interpersonal attachment style will predict identification with social groups contingent upon the presence or absence of threat to a romantic interpersonal relationship.

\section{Adult interpersonal attachment}

Adult attachment style is believed to develop from childhood relationship experiences, a functional trend that is designed to ensure predictability in future relationships (Mikulincer \& Shaver, 2007). Attachment style can be conceptualized as the combination of two continuous dimensions: attachment anxiety (fear of separation and abandonment) and attachment avoidance (discomfort with intimacy and dependency) (see Brennan, Clark, \& Shaver, 1998).1 Research has primarily focused on the implications of attachment style for romantic relationships (Shaver \& Mikulincer, 2002). Individuals higher in attachment anxiety are concerned with seeking support, acceptance, and closeness in response to their fear of rejection. In response to threats to a romantic relationship these individuals hyperactivate their attachment system, demonstrating excessive reassurance seeking (Shaver, Schachner, \& Mikulincer, 2005) and intense efforts to maintain proximity, accompanied by excessive rumination about abandonment fears (Shaver \& Mikulincer, 2002). In contrast, individuals lower in attachment anxiety are more comfortable with closeness. Such individuals report more trust ( Mikulincer, 1998b), empathy ( Mikulincer et al., 2001), cognitive openness ( Mikulincer \& Arad, 1999) and less hostile intent towards others when angry (Mikulincer, 1998a).

Orthogonal to attachment anxiety is the attachment avoidance dimension. Individuals higher in attachment avoidance deactivate the attachment system when they are exposed to threats to their relationships. Mikulincer and Shaver (2007) argue that these individuals try to garner interpersonal support while maintaining self-reliance and distance from the partner, but also try to ignore the needs that might activate the attachment system in the first place. Attachment avoidance can interact with attachment anxiety to produce unique patterns of relationship behavior. For instance, while those lower on both dimensions may be conceptualized as "secure", those higher on both may be conceptualized as "fearful avoidant" ( Bartholomew \& Shaver, 1998). Such individuals may deny support needs, but still experience anxiety and a desire for proximity with close others ( Mikulincer \& Shaver, 2007). In what follows we focus our investigation first on attachment anxiety (as it is key to the theoretical integration we propose). Later (in Experiment 2) we will further specify our model by examining a qualifying role for attachment avoidance.

\section{Identification with groups}

As well as helping us to understand how people act and react in close interpersonal relationships, attachment theory has also enabled an understanding of how people identify with others outside of romantic relationships. Of particular relevance is the finding that individuals lower in attachment anxiety seek social support beyond primary attachment figures in times of stress (e.g., friends and family; Florian, Mikulincer, \& Bucholtz, 1995). This tendency 
to sometimes look beyond primary attachment relationships for social and emotional support inspired the theoretical model we propose. In particular, we argue that sometimes these "others" may constitute not only individuals but also social groups.

Social groups are an important part of how we define ourselves (e.g., an avid football team supporter, a political party member, or a valued occupational role). Social Identity Theory (Tajfel \& Turner, 1979) argues that identification with groups is important psychologically because it satisfies basic human motives of self-verification, self-evaluation, and epistemological clarity (for a recent account see Hogg, 2006). Given the positive self-definitional properties of group identification, our initial proposition is that groups will become particularly appealing as sources of social support at times of interpersonal distress. In other words, if identification with groups serves to satisfy goals ranging from feeling positive about oneself to understanding ones' place in the world, it seems reasonable to propose they will also serve to satisfy a need to affiliate arising from interpersonal distress.

However, we believe that these consequences for ingroup identification will be qualified by individuals' attachment style. We discussed earlier how lower levels of attachment anxiety can be associated with seeking more social and emotional support beyond primary attachment figures in times of interpersonal distress (Florian et al., 1995), whereas individuals higher in attachment anxiety become fixated upon their attachment figure, with excessive proximity seeking (Shaver et al., 2005). These different behavioral tendencies should be reflected in the extent to which individuals identify with groups following interpersonal distress. Individuals lower in attachment anxiety should act as described above, identifying with groups to capitalize on the self-evaluative benefits they offer. Individuals higher in attachment anxiety, however, would not be expected to identify with groups under these conditions. These individuals tend to fixate upon their attachment figures, especially in times of distress. Therefore, rather than seeing groups as an additional source of support, they are likely to withdraw their affiliative efforts to focus on their most important attachment relationship:their romantic partner.

\section{Experiment 1}

To examine the interplay of attachment style, attachment threat, and ingroup identification we compared reactions following an envisaged relationship-threatening event. We measured both ingroup identification and behavioral action tendencies. Action tendencies are intentions related to experienced emotion and reflect the extent to which people are inclined to approach or avoid others (Mackie, Smith, \& Devos, 2000). As such, they are particularly useful for indexing relative tendencies to approach or avoid groups and individuals. We also asked participants to report interpersonal closeness and action tendencies relating to their romantic partner. This allowed us to see whether identification with groups would occur at the expense of, or independent from, closeness to romantic partner. Finally, we included a measure of experienced emotion (see Mackie et al., 2000). Consistent with the literature on adult attachment, we expected fearfulness to be associated with attachment anxiety, especially after a threat to a romantic relationship (Mikulincer \& Shaver, 2005).

\section{Methods}

\section{Participants and design}

Forty undergraduate participants (20 females, 20 males; mean age 20.6 years and in a current romantic relationship) were allocated to either the control or attachment threat condition. Attachment anxiety was measured using the Experiences in Close Relationships scale (ECR: Brennan et al., 1998). The dependent variables were identification with the participant's closest friendship group, closeness to their romantic partner, and associated action tendencies and emotions. Participants assisted on a voluntary basis.

\section{Procedure}

All participants completed the ECR. This measure has been used in many adult attachment studies and is highly reliable with both high construct and predictive validity (Shaver \& Mikulincer, 2002). Eighteen items in the ECR measure attachment anxiety. Examples of items include "I worry a lot about my relationships" and "I worry that romantic partners won't care about me as much as I care about them". Some items were coded negatively, for 
example, "I do not often worry about being abandoned". Responses were recorded on a seven point Likert-type scale of agreement anchored from 1, strongly disagree to 7 , strongly agree. The scale had good internal consistency $(\alpha=.93)$ so an index of attachment anxiety was created from the sum of all items.

Following completion of the ECR participants received either the attachment threat or control manipulation. Participants were instructed to think specifically about their partner during the threat manipulation. Participants were presented with one of two hypothetical scenarios, based on a similar method used previously by Mikulincer, Florian, Birnbaum, and Malishkevich (2002). They were instructed to read the scenario and spend three minutes imagining how it would make them feel. They were then required to write a few sentences describing these thoughts. The first scenario was attachment relevant (threat condition) involving a confrontation with their romantic partner:

While in a discussion with your boy/girlfriend about the current state of your relationship, it comes to light that he/she is not happy with the relationship as it is, and would like to spend some time apart and have some time to think. This came as a shock to you as you were under the impression things were fine and you think time apart could do more harm than good. The discussion escalates into a full-blown row and your boy/girlfriend storms out of the house slamming the door, leaving the situation unresolved. When you try to contact him/her the phone is constantly switched off. You have no means of contact.

The second scenario was an attachment neutral (control condition), although intended to be just as negative in tone:

It is a miserable Monday morning and you awake to the sound of rain outside your window. When you look at your clock you are shocked to realise that it is already 8:45 and you have a group presentation starting at 9 . You rush to get ready and because of how late you arrive, you have missed your allocated slot and may lose $30 \%$ of your module. You grab your things and leave the lecture theatre and head for home, only to realise when you arrive that in your rush to get back you have left your bag along with phone, wallet and keys on the table in the lecture theatre. When you go back in search for them you realise they have gone, but have no means of contacting your group to ask if they had seen them as you have no phone. When you get back to your house, you wait outside for an hour in the rain for your house mate to get back before you are able to get in the house. Your missing wallet contained all your cards, driving license, university I.D. and $£ 50$ worth of cash.

The control scenario was designed to invoke negative emotions to ensure that any differences in responses to the attachment threat condition were attachment related and not merely a response to an upsetting or negative situation. Following these manipulations, participants in both conditions completed the dependent measures, before being thanked, and debriefed.

\section{Dependent measures}

We included a measure of affective state with items to capture experienced emotion (following Mackie et al., 2000). Participants rated six different fearfulness-related words in terms of the extent to which they would feel these emotions following the hypothetical situation. The items were worried, anxious, afraid, fearful, troubled, uneasy ( $\alpha=$ .80). Each item was rated on a seven point Likert-type scale anchored: 1, not at all to 7, very much. 2

Identification with friendship group was measured using the Inclusion of the Other in the Self measure (IOS; Aron et al., 1992 and Tropp and Wright, 2001). The instructions were as follows: "We would like you to circle the picture below which best describes your relationship with your closest group of friends ('self' standing for you, 'group' standing for your closest group of friends)". Seven increasingly overlapping circles represented closeness to the ingroup. To measure closeness to partner, we also included a version of this measure that referred to "partner" instead of "group".

We also measured action tendencies towards the friendship group and partner. Action tendencies are the extent to which participants feel inclined to approach or avoid (in this case) their friendship group or partner. Approach in this context means the extent they would wish to spend time with their partner/group following the attachment threat 
or control condition. The items were adapted from Mackie et al. (2000) to include avoidance items as well as approach items. This was because we were seeking to explore higher identification as well as lower identification following threat, so it seemed prudent to measure both approach and avoidance. The scale included three items measuring approach tendencies. These asked participants to report the extent to which they were inclined, following the manipulation, to "spend time with", "talk to" and "be around as much as possible", referring first to their partner $(\alpha=.78)$ and then to the friendship group $(\alpha=.76)$; and three items measuring avoidance tendencies which asked participants to rate the extent to which they were inclined, following the manipulation, to "avoid", "have nothing to do with" and "keep at a distance from" their partner $(\alpha=.87)$ and then their friendship group $(\alpha=.66)$. Responses were given on a five point Likert-type scale anchored from 1, not at all to 7, very much, and items referring to the two types of tendency (approach or avoid) and two types of target (partner or friendship group) were averaged.

\section{Results and discussion}

\section{Fearfulness}

We used moderated regression analysis (Aiken \& West, 1991). Analysis of the fearfulness index revealed an interaction between attachment threat and attachment anxiety, $\beta=1.55, p=.007$, R-squared change $=.106$, see Fig. 1. Further analysis revealed that attachment anxiety was related to fearful emotions in the control condition, $\beta=$ $.457, p=.043$, and even more reliably so in the attachment threat condition, $\beta=.728, p<.0005$. This was in line with expectations. Following threat there was a strong tendency for higher levels of attachment anxiety to be associated with more fearful emotions. These emotions are strongly associated with attachment anxiety, and have been shown previously to motivate the comfort seeking and excessive reassurance behaviors exhibited by these individuals ( Feeny, 1998). This data supports the idea that fearful emotions are the trigger for the responding of individuals higher in attachment anxiety following an attachment-related threat.

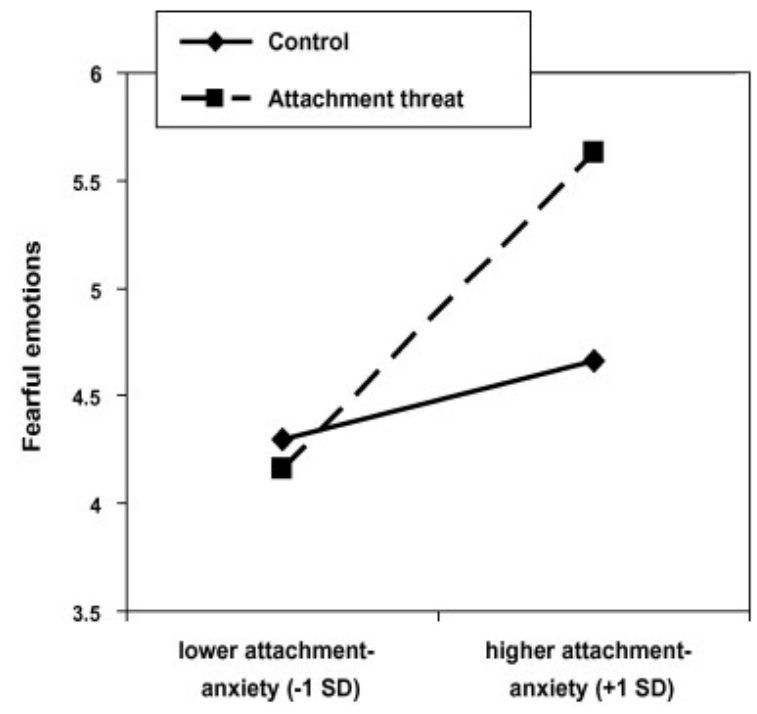

Fig. 1. - Fearful emotions as a function of attachment threat and attachment anxiety.

\section{Ingroup identification3}

The moderated regression analysis revealed a significant interaction on entering the interaction variable at step $2 ; \beta$ $=-1.84, p=.013$, R-squared change $=.150$, see Fig. 2 . Within condition analysis revealed that while attachment anxiety showed a tendency to be positively related to ingroup identification in the control condition, $\beta=.419, p=$ .066 , it was negatively related to ingroup identification in the threat condition, $\beta=-.441, p=.052$. In other words, following a threat to a romantic relationship, the higher participants' attachment anxiety, the less inclined they were to identify with groups. 


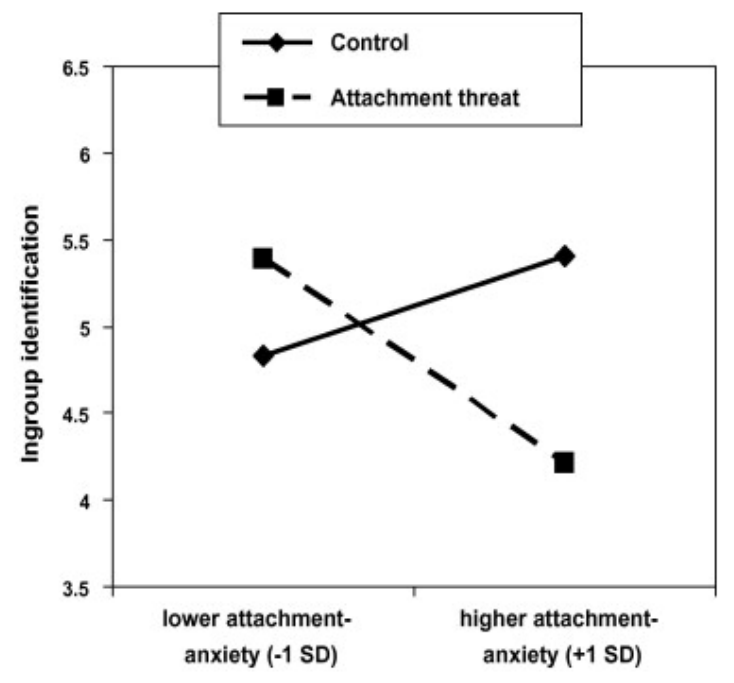

Fig. 2. -Ingroup identification as a function of attachment threat and attachment anxiety.

\section{Closeness to partner}

The moderated regression analysis revealed no interaction on entering the interaction variable at step $2 ; \beta=.254, p$ $=.653$. An attachment anxiety main effect, $\beta=.331, p=.007$, indicated that participants higher in attachment anxiety felt closer to their partner than participants lower in attachment anxiety, and this tendency was not moderated by threat. In other words, regardless of threat, attachment anxiety was positively correlated with partner closeness. This suggests that rather than being mutually exclusive, tendencies to identify with groups and to perceive closeness to attachment figures as a function of attachment anxiety are unrelated.

\section{Group approach tendency4}

We created a measure of overall tendency to approach the group by subtracting tendencies to avoid from tendencies to approach. The moderated regression analysis on the resulting index revealed a significant interaction on entering the interaction variable at step $2 ; \beta=-2.35, p=.001$, R-squared change $=.244$, see Fig. 3 . Analysis within experimental conditions revealed that while there was a trend for attachment anxiety to be positively related to approach tendencies in the control condition, $\beta=.384, p=.095$, it was negatively related to approach tendencies in the attachment threat condition, $\beta=-.634, p=.003$. In other words, higher attachment anxiety was associated with decreasing group approach tendencies. This replicated the pattern observed on the measure of ingroup identification.

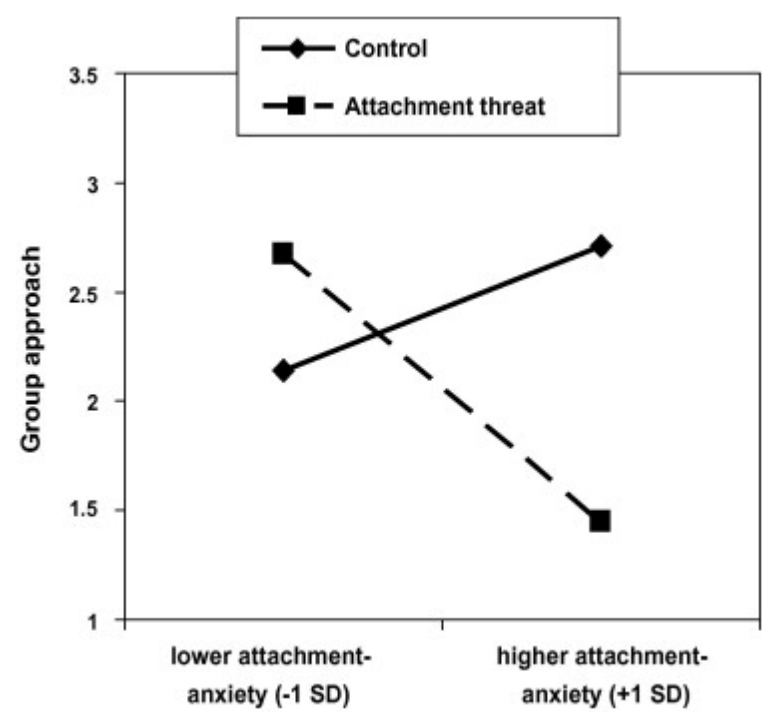

Fig. 3. - Group approach tendencies as a function of attachment threat and attachment anxiety. 


\section{Partner approach tendency}

We created a measure of overall tendency to approach the partner by subtracting tendencies to avoid from tendencies to approach. The moderated regression analysis on the resulting index revealed an attachment anxiety main effect, $\beta=.621, p<.0005$, indicating that participants higher in attachment anxiety felt a stronger tendency to want to approach their partner than participants lower in attachment anxiety. This effect was qualified, however, by a significant interaction on entering the interaction variable at step $2 ; \beta=1.25, p=.011$, R-squared change $=.069$. Analysis within conditions revealed that attachment anxiety was positively related to approach tendency in the control condition, $\beta=.472, p=.036$, and even more positively related to approach tendency in the attachment threat condition, $\beta=.823, p<.0005$. As with partner closeness, even after threat there remained a positive relationship between attachment anxiety and partner approach 5 .

Our findings show that following threat to a romantic relationship participants higher in attachment anxiety are (a) more likely to feel fearful; (b) less likely to identify with and report approach tendencies to groups. This supports our proposition that attachment anxiety will determine tendencies to see groups as offering viable sources of additional support at times of interpersonal distress. Of note, while ingroup identification and group approach tendencies were differentially related to attachment anxiety as a function of relationship threat, this was not the case for the partnerfocused measures. Regardless of threat, participants higher in attachment anxiety reported greater closeness with their partner. This is consistent with our expectations about how individuals lower in attachment anxiety will behave in response to threat. It is arguably a better strategy to identify with groups while at the same time maintaining closeness to one's partner (thus expanding the range of one's available support, yet not withdrawing unnecessarily from a potentially repairable relationship). To reject one's partner to favor one's group exclusively would be a far more risky strategy, and not consistent with the more cognizant approach of individuals lower in attachment anxiety. In other words, while relationship threat alters tendencies to identify with groups, we would not expect (and do not find) it to make any difference to feelings of closeness towards the relevant romantic partner.

\section{Experiment 2}

In Experiment 2 we extended our investigation to consider both the anxiety and avoidance dimensions of adult attachment. Because the impact of attachment avoidance is difficult to quantify with explicit self-report measures (Bartholomew \& Shaver, 1998) here we used a measure of implicit identification with groups. Individuals higher in attachment avoidance are uncomfortable with explicit expressions of interpersonal closeness, and on explicit measures we might therefore expect such participants to conceal any expression of attachment to individuals, or even identification with groups ( Fraley and Shaver, 1997, Mikulincer and Shaver, 2007 and Shaver and Mikulincer, 2004). As such, if we were to examine any interactive effects of avoidance we needed to do so using an implicit measure.

Using implicit measures we expected a moderating effect of attachment avoidance on the attachment anxiety $x$ threat effects we observed in Experiment 1. Our reasoning was as follows. Under control conditions we expected participants higher in attachment avoidance and attachment anxiety (so-called "fearful" individuals) to be those particularly inclined to look additionally to groups (as well as romantic partners) for emotional support. Attachment avoidance is associated with discomfort with interpersonal closeness, but attachment anxiety is associated with a high need for such closeness. This combination of attachment styles therefore predicts a behavioral pattern indicating a need for closeness, but a simultaneous discomfort with expression of interpersonal attachment. These apparently conflicting goals may be difficult to fulfil when focused only on individual level relationships. In contrast, satisfying a need for closeness from groups, rather than individuals, may offer the solution to the dilemma that characterizes these individuals. Groups can be supportive, yet limited in the level of intimacy they provide (or require in return). As such they may be a good option for avoidant individuals. We therefore expected to observe a strengthening of the trend observed in Experiment 1: a positive relationship between attachment anxiety and group identification under control conditions. This prediction translates into an attachment anxiety $\times$ attachment avoidance interaction in the control condition, where the positive relationship between attachment anxiety and identification with groups will be most apparent for participants higher in attachment avoidance. In contrast, under 
relationship threat, the moderating effect of avoidance should not emerge. As we observed in Experiment 1, following relationship threat group identification is no longer emotionally viable for individuals higher in attachment anxiety, thus removing the possible "safe" option for individuals higher in attachment avoidance. As such, we should replicate the observed negative relationship between attachment anxiety and identification following threat irrespective of the level of attachment avoidance.

In this experiment we also compared different types of target group. Specifically, we examined identification with groups low and high in perceived entitativity. Entitativity is the extent to which a collection of individuals are perceived as a coherent group. According to Lickel et al. (2000) four types of group exist (intimacy groups, task groups, social categories and loose associations) which differ in their level of entitativity. A friendship group (as measured in Experiment 1) can be classified as an intimacy group and therefore high in entitativity, while a broader social categorization, like gender, can be considered low in entitativity ( Lickel et al., 2000). Given the argument we outlined above, that groups will be particularly appealing for individuals higher in attachment avoidance and anxiety because they require less intimacy, we may expect the effects of attachment avoidance to be contingent upon the extent to which the group itself is characterized by higher or lower levels of intimacy. If groups are appealing for these individuals because they offer "safe" (non-intimate) affiliations, we might expect less entitative groups to be most appealing. Put another way, it could be that less entitative groups are precisely those groups that appeal to individuals higher in attachment avoidance and anxiety because they offer the desired support but require less intimacy than more entitative groups.

\section{Methods}

\section{Participants and design}

One-hundred and twelve female psychology students (mean age 19.8) were allocated to one of two conditions (control vs. attachment threat) and one of two Implicit Association Tests (IAT: Greenwald, McGhee, \& Schwartz, 1998) referring to either gender categories or friendship groups.6 Participants received either course credit or monetary payment.

\section{Procedure}

After providing all necessary self-relevant information (see below) participants completed the ECR, followed by the control versus threat manipulation used in Experiment 1. The correlation between the attachment anxiety $(a=.92)$ and attachment avoidance $(a=.95)$ scales was $r(112)=-.077, p=.423$. After this participants completed the IAT. The IAT task focused on either the strength of the association between self and friends (identification with friendship group) or the strength of the association between self and gender (identification with gender group). At the end of the experiment participants were thanked and debriefed.

\section{Dependent measures}

The IAT is an experimental procedure which is used to test the implicit strength of associations between categories. The basic methodology of the IAT entails the categorization of words or pictures presented on a computer screen. The idea is that when categorizing along two dimensions simultaneously then implicit associations between concepts tested will be reflected in response times. Specifically, when participants are required to categorize two words (e.g., an ingroup name and a positive word) and this response requires pressing the same response key, then this will be easier for participants than when different response keys are required (e.g., an ingroup name and a negative word). The result of this will be that faster response times will be observed on trials representing hypothesized strong associative links (ingroup-positive and outgroup-negative) compared to trials representing weak associative links (i.e., ingroup-negative and outgroup-positive). Implicit association is defined as the extent to which congruent pairings are responded to faster than incongruent pairing (the "IAT effect").

\section{IAT construction}

Both the friendship group IAT and the gender IAT required stimuli referring to the self. To provide these stimuli, participants were asked, prior to the ECR, to answer eight questions relating to themselves ("me", e.g. hometown, 
family name etc.) and eight corresponding questions that did not relate to themselves ("not me"). Participants were informed that the information they supplied would be used in the task that followed (the listed stimulus words were inputted into the computer program). It was important to create a set of questions which were representative of the self, but did not consist of any attachment-related information (e.g., surname). Example questions included: "Write your birthday in the space provided. For example write 22nd March", "Write your hometown in the space provided". Participants were then required to answer the corresponding "not me" questions, choosing relevant answers from a word list. Choices were made based on the criteria that the participants were familiar with the item, the item was not associated or identified with them, and that they neither liked nor disliked the item (Greenwald, 2003).

\section{Friendship group IAT}

In order to create the friendship group IAT, prior to the ECR, participants were given the following instructions:

Please state the first names of eight of your female friends. The names do not have to be in any particular order. The list can be made up of friends you know from any area of your life, for example the list can include friends from university, school, home, work, sports team, etc.

Eight numbered spaces then followed. The names listed formed the "friend" element of the IAT. In order to create the "not friend" element, participants were given the following instructions:

Using the list below, please circle eight names which fit the following criteria:

1. You are familiar with the name, i.e. you have heard the name before;

2. You do not associate the name with anybody, i.e. it is NOT the name of anybody specific (a person you know, celebrity, TV character, etc.).

A list of 125 names then followed which were compiled from the National Statistics top 100 girls names in England and Wales for the years 2002-2006 (with repeated names omitted). These popular names were used in order to fulfil the IAT criterion that participants should be familiar with the items (i.e., be familiar with the names). Each participant received the names in a randomized order.

\section{Gender IAT}

The gender IAT required female ("me") and male ("not me") stimulus items. A list of eight female names (Jessica; Lucy; Emily; Sophie; Hannah; Emma; Abigail; Charlotte) and eight male names (William; Jack; James; Thomas; Daniel; Luke; Matthew; Alexander) were used (names taken from the top 20, National Statistics, 2003; see Rosenthal, 2006). Names were matched for letter length, and unisex names were excluded.

\section{Results and discussion}

We used the latest scoring algorithm for the IAT to create D-score measures of implicit association (Greenwald, Nosek, \& Banaji, 2003). We computed a moderated regression analysis including four factors: target group (friends vs. gender), threat (control vs. attachment threat), attachment anxiety (continuous lower to higher) and attachment avoidance (continuous lower to higher). This analysis yielded the predicted four-way interaction, $\beta=-1.68, p=.028$, $\mathrm{R}$-squared change $=.019$. However, despite a tendency showing the threat $\times$ avoidance $\times$ anxiety interaction to be stronger for gender groups than friendship groups (consistent with the idea that higher attachment avoidance will make less intimate, more abstract groups preferable) there were no clear differences in the pattern of lower order interactions between target groups. The analysis collapsed across gender and friendship groups, however, did yield an interpretable pattern of results (the lower order threat $x$ anxiety $\times$ avoidance interaction was highly significant, $\beta$ $=2.82, p=.005$, R-squared change $=.068$ ). We therefore focus on this and discuss the weaker evidence for moderation by target group entitativity in the general discussion.

To decompose the threat $x$ anxiety $\times$ avoidance interaction we carried out separate anxiety $\times$ avoidance moderated regression analyses in the control and attachment threat conditions. In the control condition this analysis revealed a significant anxiety $\times$ avoidance interaction, $\beta=-1.47, p=.005$ but no other significant effects. Further decomposition 
revealed that, in the control condition, attachment anxiety was positively related to implicit group identification for participants higher in attachment avoidance, $\beta=1.16, p=.011$, but this relationship was weaker for participants lower in attachment avoidance, $\beta=0.44, p=.052$. This is consistent with our predictions: it is those participants who are interpersonally avoidant, when also higher in attachment anxiety, who are more likely to seek groups as a source of support. These participants can be described as "fearful" (i.e., both high avoidance and high anxiety) and this supports other research which has found that fearfully attached participants are more likely to seek support than those who are high on avoidance but low on anxiety (i.e., dismissive) ( Allen \& Baucom, 2004). We argue that groups can be considered "safer" than interpersonal relationships, where it is possible to garner emotional support without having to offer as much intimacy in exchange. This also qualifies our finding in previous studies that anxiety and group identification are typically positively related under control conditions. This seems to be especially the case for higher avoidance participants, at least at the implicit level.

In contrast to the moderating role of attachment avoidance in the control condition, in the attachment threat condition there was no anxiety $\times$ avoidance interaction and only a negative relationship between anxiety and implicit group identification, $\beta=-2.89, p=.033$. This replicates, at an implicit level, the relationship between anxiety and identification under threat observed in Experiment 1. Following attachment threat groups are no longer a desirable option for individuals higher in attachment anxiety. Correspondingly, there is no impact here of the strategy of individuals higher in avoidance to use groups as an alternative (low intimacy) source of support.

\section{General discussion}

In this research we predicted that attachment anxiety would determine whether and when individuals would turn to social groups as additional sources of support at times of interpersonal distress. In two studies we observed evidence consistent with these predictions. Higher attachment anxiety was associated with withdrawal from groups (lower ingroup identification) and greater closeness with relationship partners. This was the case whether the group in question was a friendship group or social category. In contrast, at lower levels of attachment anxiety participants tended to additionally identify with groups (whether a friendship group or social category), although not at the expense of interpersonal closeness to partners.

We also observed these tendencies on measures of both explicit and implicit identification with groups, and we have shown that while attachment avoidance moderates the relationship between attachment anxiety and identification under control conditions, under attachment threat higher attachment anxiety is associated with lower identification with groups irrespective of attachment avoidance. Below we discuss the implications of these affiliative tendencies in response to attachment threat.

\section{Implications and future work}

To our knowledge this is the first research to explore the relationship between interpersonal attachment and identification with groups following an interpersonally-focused threat. Under control conditions participants' attachment anxiety was positively correlated with ingroup identification. This finding fits with the theory that anxiously attached individuals hyperactivate their attachment systems and demonstrate a need for closeness within relationships (Shaver et al., 2005)-including relationships with groups-and that this tendency is especially the case for individuals higher in attachment avoidance, for whom groups offer an ideal "safe" (low intimacy) source of support.

The effects observed can be partly explained by the experience of fearful emotions following the threat. Within Mikulincer and Shaver's three-phase model of attachment-system activation and dynamics, a threat is perceived, the attachment figure's availability and responsiveness is assessed, and if unavailability is perceived, either hyperactivating or deactivating strategies are employed depending on whether proximity seeking is a viable protective strategy (Shaver \& Mikulincer, 2002). In this model, individuals higher in attachment anxiety would be highly sensitive to threat related cues, and would typically use hyperactivating strategies following perceived unavailability (Mikulincer \& Shaver, 2005). Our research supports these arguments, demonstrating that in times of 
relationship threat, higher attachment anxiety is associated with both fearful emotions and lower identification with groups. The finding that higher attachment anxiety is associated with lower identification with groups, but not the attachment figure appears to support our argument that for such individuals, in the context of threat, all other extraneous relationships are temporarily forgone in favor of the primary attachment figure.

Lower attachment anxiety was associated with heightened identification with groups following threat. We argue that this indicates an adaptive strategy both cognitively and behaviorally:the seeking of additional sources of support when needed. This finding parallels other research which suggests that securely attached persons demonstrate adaptive responses to threatening situations, for example by seeking support to cope with distress (Shaver \& Mikulincer, 2004). Cognitively, attending to identification with other groups may represent a way for these participants to divert attention away from the attachment threat until the threat has been resolved, thus avoiding hyperactivation of the attachment system and the spread of negative emotions.

In Experiment 2 we observed some evidence that the relationships observed in Experiment 1 are more applicable when the groups involved are lower in entitativity (e.g., social categories like gender) compared to those higher in entitativity (e.g., intimacy groups like friendship groups). This is consistent with the idea that attachment avoidance (discomfort with interpersonal closeness) will make more abstract, less intimate groups preferable for additional sources of support when required (i.e., when individuals are also higher in attachment anxiety). We note, however, that for both types of target group the threat $\times$ avoidance $\times$ anxiety effect was significant, so we remain tentative about the apparent effects of target entitativity (and implied intimacy), and this remains an important focus for future work.

\section{Conclusions}

Previous work has collectively made a step towards establishing attachment theory as a useful tool for explaining group processes. There has, however, been little focus to date on the impact of dyadic attachment style on an individual's propensity to identify with groups, or how this may vary when the attachment system is activated in the context of threat. In this research we tested the idea that when people experience threat to their close relationships then attachment anxiety will predict whether and when they identify with groups. We observed evidence supportive of the idea that following attachment threat higher attachment anxiety is associated with lower identification with groups while lower attachment anxiety is associated with higher identification with groups. These findings suggest some intriguing possibilities about the link between attachment to individuals and identification with groups and support the idea that integration of diverse literatures in group processes, interpersonal attachment, and social identification can enhance our understanding of the nature of social relationships.

\section{References}

L.S. Aiken, S.G. West

Multiple regression: Testing and interpreting interactions

Sage, London (1991)

\section{E.S. Allen, D.H. Baucom}

Adult attachment and patterns of extradyadic involvement

Family Processes, 43 (2004), pp. 467-488

A. Aron, En. Aron, D. Smollan

Inclusion of other in the self scale and the structure of interpersonal closeness

Journal of Personality and Social Psychology, 63 (1992), pp. 596-612

K. Bartholomew, P.R. Shaver

Methods of assessing adult attachment: Do they converge?

J.A. Simpson, S. Rholes (Eds.), Attachment theory and close relationships, Guilford Press, New York (1998), pp. 25-45

K.A. Brennan, C.L. Clark, P.R. Shaver

Self-report measurement of adult attachment: An integrative overview 
J. Feeny

Relationship centred anxiety

J.A. Simpson, W.S. Rholes (Eds.), Attachment theory and close relationships, Guilford Press, New York (1998), pp. 46-76

V. Florian, M. Mikulincer, I. Bucholtz

Effects of adult attachment style on the perception and search for social support

Journal of Psychology, 129 (1995), pp. 665-676

\section{R.C. Fraley, P.R. Shaver}

Adult attachment and the suppression of unwanted thoughts

Journal of Personality and Social Psychology, 73 (1997), pp. 1080-1091

L. Gaertner, J. Schopler

Perceived ingroup entitativity and intergroup bias: An interconnection of self and others

European Journal of Social Psychology, 28 (1998), pp. 963-980

Greenwald, A. G. (2003). Implicit association test materials. Retrieved September 4, 2004 from http://faculty.washington.edu/agg/iat_materials.htm.

\section{A.G. Greenwald, D.E. McGhee, J.L.K. Schwartz}

Measuring individual differences in implicit cognition: The implicit association test

Journal of Personality and Social Psychology, 74 (1998), pp. 1464-1480

A.G. Greenwald, B.A. Nosek, M.R. Banaji

Understanding and using the implicit association test: I. An improved scoring algorithm

Journal of Personality and Social Psychology, 85 (2003), pp. 197-216

\section{M.A. Hogg}

Social identity theory

P.J. Burke (Ed.), Contemporary social psychological theories, Stanford University Press, Palo Alto, CA (2006), pp. 111-136

B. Lickel, D.L. Hamilton, G. Wieczorkowska, A. Lewis, S.J. Sherman, A.N. Uhles

Varieties of groups and the perception of group entitativity

Journal of Personality and Social Psychology, 78 (2000), pp. 223-246

D.M. Mackie, T. Devos, E.R. Smith

Intergroup emotions: Explaining offensive action tendencies in an intergroup context

Journal of Personality and Social Psychology, 79 (2000), pp. 602-616

M. Mikulincer

Adult attachment style and individual differences in functional versus dysfunctional experiences of anger

Journal of Personality and Social Psychology, 74 (1998), pp. 513-524

M. Mikulincer

Attachment working models and the sense of trust: An exploration of interaction goals and affect regulation

Journal of Personality and Social Psychology, 74 (1998), pp. 1209-1224

M. Mikulincer, D. Arad

Attachment working models and cognitive openness in close relationships: A test of chronic and temporary accessibility effects Journal of Personality and Social Psychology, 77 (1999), pp. 710-725

M. Mikulincer, V. Florian, G. Birnbaum, S. Malishkevich

The death-anxiety buffering function of close relationships: Exploring the effects of separation reminders on death-thought accessibility Personality and Social Psychology Bulletin, 28 (2002), pp. 287-299

M. Mikulincer, O. Gillath, V. Halevy, N. Avihou, S. Avidan, N. Eshkoli

Attachment theory and reactions to others needs: Evidence that activation of the sense of attachment security promotes empathic responses Journal of Personality and Social Psychology, 81 (2001), pp. 1205-1224 
M. Mikulincer, G. Hirschberger, O. Nachmias, O. Gillath

The affective component of the secure base schema: Affective priming with representations of attachment security

Journal of Personality and Social Psychology, 81 (2001), pp. 305-321

M. Mikulincer, P.R. Shaver

Attachment theory and intergroup bias: Evidence that priming the secure base schema attenuates negative reactions to out-groups Journal of Personality and Social Psychology, 81 (2001), pp. 97-115

M. Mikulincer, P.R. Shaver

Attachment theory and emotions in close relationships: Exploring the attachment-related dynamics of emotional reactions to relational events Personal Relationships, 12 (2005), pp. 149-168

M. Mikulincer, P.R. Shaver

Attachment in adulthood: Structure, dynamics, and change

Guilford Press (2007)

Rosenthal, H. E. S. (2006). Reducing stereotype threat: A category differentiation approach. Unpublished doctoral dissertation, University of Birmingham, UK.

P.R. Shaver, M. Mikulincer

Attachment-related psychodynamics

Attachment and human development, 4 (2002), pp. 133-161

P.R. Shaver, M. Mikulincer

What do self-report attachment measures assess?

W.S. Rholes, J.A. Simpson (Eds.), Adult attachment: Theory, research and clinical implications, Guilford Press, London (2004), pp. 17-54

P.R. Shaver, D.A. Schachner, M. Mikulincer

Attachment style, excessive reassurance seeking, relationship processes, and depression

Personality and Social Psychology Buletin, 31 (2005), pp. 343-359

E.R. Smith, J. Murphy, S. Coates

Attachment to groups: Theory and measurement

Journal of Personality and Social Psychology, 77 (1999), pp. 94-110

Tajfel, H., \& Turner, J. C. (1979). An integrative theory of intergroup conflict. In S. Worchel \& W. G. Austin (Eds.).

\section{L.R. Tropp, S.C. Wright}

Ingroup identification as the inclusion of ingroup in the self

Personality and Social Psychology Bulletin, 27 (2001), pp. 585-600

1. Although we define attachment styles here as continua, we also acknowledge that it is possible for behaviours associated with particular styles to be contextually primed. For instance, priming the secure base schema leads to more positive affective reactions, independent of chronically-defined attachment style (Mikulincer, Hirschberger, Nachmias, \& Gillath, 2001).

2. We also measured anger and happiness items but because they hold no particular theoretical interest for our hypotheses we do not report them further.

3. A higher order moderated regression including attachment anxiety (continuously lower to higher), threat (control vs. attachment threat) and target (group vs. partner) revealed a significant interaction on entering the interaction variable at step $2 ; b=-1.39, p=.021$, R-squared change $=.086$. This indicated the presence of a three-way interaction between threat, attachment anxiety and target (group vs. partner). We report the decomposition analyses for group (identification) and partner (closeness) independently in the main text.

4. As with identification and partner closeness we also computed a moderated regression including target as a withinsubjects variable (group vs. partner). However, we also divided the action tendencies up into two separate components, approach and avoidance tendencies, which provided an additional within-subjects factor. We therefore calculated a moderated regression including attachment anxiety (continuously lower to higher), threat (control vs. 
attachment threat), target (group vs. partner) and tendency (approach vs. avoidance), with the last two factors withinsubjects. This analysis revealed a significant four way interaction on entering the interaction variable at step $2 ; b=$ $-2.23, \mathrm{p}<.0005$, R-squared change $=.221$. We decomposed this interaction by carrying out separate threat $x$ attachment anxiety interactions for action tendencies for group and partner targets, respectively. To simplify the analysis we created a composite index of approach tendency (approach tendency minus avoidance tendency) and computed the above analysis on this index for partner and group targets, respectively, reported in the main text.

5. Consistent with existing findings from the adult attachment literature, we found that fearful emotions mediated the relationship between attachment anxiety and partner closeness following threat $(Z=2.84, p=.005)$. The role of fearful emotions in driving tendencies to identify with groups is more complex. Here it is the absence of these fearful emotions that provides the opportunity for those lower in attachment anxiety to maintain a strategic identification with groups. As such, we would not expect fearful emotions to mediate the negative relationship between attachment anxiety and ingroup identification, but we might expect it to suppress this relationship. In other words, if the absence of fearful emotions promotes identification with groups, entering it as a co-variate should strengthen the attachment anxietyidentification relationship. In step 1, the outcome measure (group approach as a proxy for identification) was regressed onto the predictor (attachment anxiety) revealing a significant negative relationship, $\beta=-.634, p=.003$. In step 2, attachment anxiety predicted the mediator (fearful emotions), $\beta=.728, p<.0005$. In step 3 , group approach was regressed onto fearful emotions while controlling for attachment anxiety, which also revealed a significant relationship, $\beta=.621, p=.015$. Finally, when the mediator was controlled, the relationship between attachment anxiety and group approach became more significant, $\beta=-1.09, p<.0005$ (indicating that fearful emotions, for group approach behaviours, is a suppressor). A Sobel test was significant, $Z=2.33, p=.02$.

6. A pre-test confirmed that the gender and friendship groups were low and high entitativity as predicted. Twenty-four male $(N=12)$ and female $(N=12)$ undergraduate students (mean age $=24.7 ; S D=3.1$ ) were asked to complete entitativity measures outlined by Lickel et al., 2000 and Gaertner and Schopler, 1998. Participants were asked, respectively, to 'please rate the extent to which each one qualifies as a group', ranging from 1 (not a group at all) to 9 (very much a group) and to select which of a set of six diagrams best represented the relevant group (each diagram contained five circles that became closer until they were contained within one unit). The order of group presentation was counterbalanced. On the Lickel et al. measure the gender group ( $M=5.13)$ was rated lower in entitativity than the friendship group $(M=7.75), t(23)=-4.04, p=.001$. On the Gaertner and Schopler scale this was also the case, the gender group $(M=2.50)$ was rated as lower in entitativity compared to the friendship group $(M=4.89), t(23)=-6.42, p$ $<.001$. 